MZ-TH/93-32

November 1993

\title{
Inclusive Semileptonic B Decays in QCD including Lepton Mass Effects
}

\author{
S. Balk ${ }^{1}$ \\ J.G. Körner ${ }^{2}$ \\ D. Pirjol ${ }^{1}$ \\ and \\ K. Schilcher ${ }^{2}$ \\ Johannes Gutenberg-Universität \\ Institut für Physik, Staudingerweg 7 \\ D-55099 Mainz, Germany
}

\begin{abstract}
Starting from an Operator Product Expansion in the Heavy Quark Effective Theory up to order $1 / m_{b}^{2}$ we calculate the inclusive semileptonic decays of unpolarized bottom hadrons including lepton mass effects. We calculate the differential decay spectra $d \Gamma /\left(d E_{\tau}\right)$, and the total decay rate for $\mathrm{B}$ meson decays to final states containing a $\tau$ lepton.
\end{abstract}

\footnotetext{
${ }^{1}$ Supported by the Graduiertenkolleg Teilchenphysik, Universität Mainz

${ }^{2}$ Supported in part by the BMFT, FRG under contract 06MZ730
} 


\section{Introduction}

Recently, the theoretical investigation of inclusive semileptonic bottom hadron $\left(H_{b}\right)$ decays has witnessed a renaissance through the use of the Operator Product Expansion (OPE) in the Heavy Quark Effective Theory (HQET) [1], 5]. Up to now these investigations have been limited to the discussion of the zero lepton mass case. While this is a good approximation for inclusive semileptonic $H_{b}$ decays involving an electron or a muon, a study of inclusive semileptonic $H_{b}$ decays involving a $\tau$ must certainly include the discussion of lepton mass effects.

There are always two aspects to the lepton mass problem in semileptonic decays. First the decay dynamics changes as one is probing also the spin zero components of the exchanged gauge boson in addition to the spin one components active in the zero lepton mass case. In the formalism of semileptonic inclusive decays this means that there are two more structure functions $\left(W_{4}, W_{5}\right)$ contributing to the decay as compared to the zero lepton mass case $\left(W_{1}, W_{2}, W_{3}\right)$. In addition the decay kinematics gets changed and becomes somewhat more complicated than in the zero lepton mass case. As the very dynamics of the OPE method entangles the decay kinematics of particle and parton decay in a subtle manner one needs to carefully discuss the decay kinematics of the massive lepton case.

Apart from theoretical considerations the calculation of inclusive semileptonic bottom decays has immediate phenomenological implications since current high energy experiments have reached a stage of sophistication that allow them to start measuring inclusive semileptonic $H_{b}$ rates involving the $\tau$. For example, recently the ALEPH collaboration reported on the first measurement of the inclusive semileptonic bottom hadron decay $H_{b} \rightarrow X \tau^{-} \bar{\nu}_{\tau}$ [ [6]. They quote a branching ratio

$$
\operatorname{Br}\left(H_{b} \rightarrow X \tau \bar{\nu}_{\tau}\right)=(2.76 \pm 0.47 \pm 0.43) \% .
$$

In this presentation we shall closely follow the notation of [4], albeit with the necessary extensions to include lepton mass effects. We also include a separate section on the operator product method in HQET where we present

general formulae which allow one to go beyond the $1 / m_{b}^{2}$ results considered in [2, 5]. 


\section{Operator Product Expansion in HQET}

Inclusive semi-leptonic B-decays are related to the transition operator

$$
t_{\mu \nu}=-i \int d^{4} x e^{-i q x} T \bar{b}(x) \Gamma_{\mu} q(x) \bar{q}(0) \Gamma_{\nu} b(0),
$$

where $q(x)$ stands for up or charm quarks. The propagator term in (2) can be written in momentum space as

$$
\stackrel{q(x) \bar{q}(0)}{=}=i S(x, 0)=i \int \frac{d^{4} l}{(2 \pi)^{4}} e^{-i l x} S(l) .
$$

The up or charm quarks may be considered as propagating in the external gluon field $F_{\alpha \beta}$ generated by the heavy quark source. The expansion of the quark propagator in powers of the external field is best derived in the FockSchwinger gauge following the general discussion in [7]. One obtains

$$
\begin{aligned}
S(l)= & {\left[\frac{1}{\not \ell-m}+\frac{1}{\not l-m} \gamma^{\alpha} \frac{1}{\not-m} \gamma^{\beta} \frac{1}{\not-m}\left(\frac{-i g}{2}\right) F_{\alpha \beta}\right.} \\
& \left.+\frac{g}{3}\left(D_{\beta} F_{\gamma \alpha}+D_{\gamma} F_{\beta \alpha}\right) \frac{1}{\not l-m} \gamma^{\alpha} \frac{1}{\not l-m} \gamma^{\beta} \frac{1}{\not l-m} \gamma^{\gamma} \frac{1}{\not l-m}+\ldots\right],
\end{aligned}
$$

where $D_{\alpha}=\partial_{\alpha}+i g A_{\alpha}^{a} t^{a}$.

The OPE can only be meaningfully applied to (2) if short distance (large momentum) contributions are absorbed in the Wilson coefficients and the long distance (small momentum) contributions are absorbed in the matrix elements of the operators. We must explicitly extract the large mass scale into an exponential, i.e. pass to the operators in HQET. We write

$$
b(x)=e^{-i m_{b} v \cdot x} B(x) .
$$

The field $B(x)$ is related to the usual field $b_{v}(x)$ of the HQET by 8

$$
B(x)=\left[1+\frac{i \not D_{\perp}}{2 m_{b}}+\frac{1}{4 m_{b}^{2}}\left(v \cdot D \not D_{\perp}-\frac{1}{2} \not D_{\perp}^{2}\right)\right] b_{v}(x),
$$

where $D_{\perp}^{\mu}=D^{\mu}-v^{\mu} v \cdot D$. Using the expansion

$$
\begin{aligned}
B(x) & =B(0)+(x \cdot D) B(0)+\frac{1}{2}(x \cdot D)^{2} B(0)+\ldots \\
& =e^{-i k x} B(0)
\end{aligned}
$$


where $k_{\mu}=i D_{\mu}$ relates to the residual momentum of the heavy quark, we obtain from (2)

$$
t_{\mu \nu}=\int \frac{d^{4} l}{(2 \pi)^{4}} \int d^{4} x e^{-i x\left(q-m_{b} v+l-k\right)} \cdot \bar{B}(0) \Gamma_{\mu} S(l) \Gamma_{\nu} B(0) .
$$

The integrations in (8) are trivially carried out with the result

$$
t_{\mu \nu}=B(0) \Gamma_{\mu} S\left(m_{b} v+k-q\right) \Gamma_{\nu} B(0) .
$$

To obtain explicit results from this master formula we must substitute for the

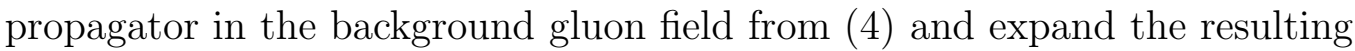
expressions in powers of $k$ using the formula

$$
\begin{aligned}
\frac{1}{\not p+\not k-m}= & \frac{1}{\not p-m}-\frac{1}{\not p-m} \not k \frac{1}{\not p-m} \\
& +\frac{1}{\not p-m} \not k \frac{1}{\not p-m} \not k \frac{1}{\not p-m}-\ldots+\ldots,
\end{aligned}
$$

where $p=m_{b} v-q$.

\section{$3 \quad$ Inclusive Decays $H_{b} \rightarrow X_{c, u}+\tau+\bar{\nu}_{\tau}$}

Consider a heavy hadron $H_{b}$ moving with a four-velocity $v$ and having a mass $M_{H_{b}}$ (containing a b quark of mass $m_{b}$ ) which decays weakly into a hadronic system of invariant mass $M_{X}$ (containing a c or u quark of mass $m_{j}(j=\mathrm{c}, \mathrm{u})$ ) and an off-shell $W^{-}$with momentum $q$. The $W^{-}$decays subsequently into a $\tau$-lepton and an antineutrino $\bar{\nu}_{\tau}$. Previous authors have investigated inclusive semileptonic $H_{b}$ decays involving the $e$ - and $\mu$-lepton where the mass of the final lepton can be neglected [2]-[5]. We extend the previous work to the case of the tau lepton where the $\tau$-mass $\left(m_{\tau}=1.777 \mathrm{GeV}\right)$ cannot be neglected. We denote the energies of the antineutrino and tau in the rest-frame of the initial hadron by $E_{\nu}$ and $E_{\tau}$, respectively.

The phase space in the variables $\left(q^{2}, E_{\tau}\right)$ has the form shown in Fig.1 for the inclusive decay $B \rightarrow X_{c} \tau \bar{\nu}_{\tau}$ and in Fig.2 for the decay $B \rightarrow X_{u} \tau \bar{\nu}_{\tau}$. $E_{\tau}$ may take values from $m_{\tau}$ up to $\left(M_{H_{b}}^{2}-\left(M_{X_{m i n}}+m_{\tau}\right)^{2}\right) /\left(2 M_{H_{b}}\right)$ and $q^{2}$ is 
bounded by $[9$

$$
m_{\tau}^{2} \leq q^{2} \leq \frac{M_{H_{b}}\left(M_{H_{b}}^{2}-M_{X_{m i n}}^{2}+m_{\tau}^{2}-2 M_{H_{b}} E_{\tau}\right)\left(E_{\tau}+\sqrt{E_{\tau}^{2}-m_{\tau}^{2}}\right)+m_{\tau}^{2} M_{X_{m i n}}^{2}}{M_{H_{b}}^{2}+m_{\tau}^{2}-2 M_{H_{b}} E_{\tau}}
$$

Here $M_{X_{\text {min }}}=m_{D}$ for $b \rightarrow c$ and $=m_{\pi}$ for $b \rightarrow u$.

The hadronic tensor of the inclusive process under consideration is defined to be

$$
W_{j}^{\mu \nu}=(2 \pi)^{3} \sum_{X} \delta^{4}\left(p_{H_{b}}-q-p_{X}\right)\left\langle H_{b}(v, s)\left|J_{j}^{\dagger \mu}\right| X\right\rangle\left\langle X\left|J_{j}^{\nu}\right| H_{b}(v, s)\right\rangle,(12
$$

where $J_{j}^{\mu}$ is the hadronic current and $j=u, c$. The expansion of $W^{\mu \nu}$ in terms of Lorentz invariant structure functions reads

$$
W^{\mu \nu}=-g^{\mu \nu} W_{1}+v^{\mu} v^{\nu} W_{2}-i \epsilon^{\mu \nu \rho \sigma} v_{\rho} q_{\sigma} W_{3}+q^{\mu} q^{\nu} W_{4}+\left(q^{\mu} v^{\nu}+v^{\mu} q^{\nu}\right) W_{5}
$$

where $q=p_{\tau}+p_{\nu}$ and $W_{1}, . ., W_{5}$ are the structure functions to be determined from the dynamics. Note that there is no T-odd term proportional to $\left(q^{\mu} v^{\nu}-\right.$ $v^{\mu} q^{\nu}$ ) in the fully inclusive decay.

The contraction of the hadronic with the leptonic tensor gives the spin averaged differential decay rate

$$
\begin{aligned}
\frac{d \Gamma}{d q^{2} d E_{\tau} d E_{\nu}}= & \frac{\left|V_{j b}\right|^{2} G_{F}^{2}}{2 \pi^{3}}\left(W_{1}\left(q^{2}-m_{\tau}^{2}\right)+W_{2}\left(2 E_{\tau} E_{\nu}-\frac{q^{2}-m_{\tau}^{2}}{2}\right)\right. \\
& +W_{3}\left(q^{2}\left(E_{\tau}-E_{\nu}\right)-m_{\tau}^{2}\left(E_{\nu}+E_{\tau}\right)\right) \\
& \left.+W_{4}\left(\frac{m_{\tau}^{2}}{2}\left(q^{2}-m_{\tau}^{2}\right)\right)+W_{5}\left(2 m_{\tau}^{2} E_{\nu}\right)\right) .
\end{aligned}
$$

In comparison to the massless lepton case the factors which multiply $W_{1}$ to $W_{3}$ change, and there are additional contributions from the structure functions $W_{4}$ and $W_{5}$.

The structure functions $W_{1}, \ldots, W_{5}$ are given by the absorptive part of the matrix element of the transition operator

$$
\begin{aligned}
T^{\mu \nu} & =\left\langle H_{b}\left|t_{\mu \nu}\right| H_{b}\right\rangle \\
& =-g^{\mu \nu} T_{1}+v^{\mu} v^{\nu} T_{2}-i \epsilon^{\mu \nu \rho \sigma} v_{\rho} q_{\sigma} T_{3}+q^{\mu} q^{\nu} T_{4}+\left(q^{\mu} v^{\nu}+v^{\mu} q^{\nu}\right) T_{5},
\end{aligned}
$$


where $\operatorname{Im} T^{\mu \nu}=-\pi W^{\mu \nu}$. What remains to be done is to determine the amplitudes $T_{1}, . ., T_{5}$.

First we expand the master formula (9) of our operator product expansion in powers of $k$. As we are interested only in operators up to dimension five we can terminate the expansion at order $k^{2}$. From the expansion of

$$
\frac{\bar{u} \gamma^{\mu}\left(1-\gamma_{5}\right)\left(m_{b} \not \gamma-\not h+\not k+m_{j}\right) \gamma^{\nu}\left(1-\gamma_{5}\right) u}{4\left(\left(m_{b} v-q+k\right)^{2}-m_{j}^{2}+i \epsilon\right)},
$$

in powers of $k$ we obtain the contribution of the $k^{0}$ - terms to $T^{\mu \nu}$ :

$$
\begin{aligned}
T_{1}^{(0)} & =\frac{m_{b}-v \cdot q}{2 \Delta_{0}} \\
T_{2}^{(0)} & =\frac{m_{b}}{\Delta_{0}} \\
T_{3}^{(0)} & =\frac{1}{2 \Delta_{0}} \\
T_{4}^{(0)} & =0 \\
T_{5}^{(0)} & =\frac{-1}{2 \Delta_{0}} .
\end{aligned}
$$

The $T_{1}^{(i)}, T_{2}^{(i)}, T_{3}^{(i)}$ terms were already given in 4 . We list them here only for completeness. The contributions of the $k^{1}$ - terms to $T^{\mu \nu}$ read:

$$
\begin{aligned}
& T_{1}^{(1)}=m_{b} E_{b}\left(\frac{1}{2 \Delta_{0}}-\frac{\left(m_{b}-v \cdot q\right)^{2}}{\Delta_{0}^{2}}\right)+\frac{2 m_{b}}{3}\left(K_{b}+G_{b}\right)\left(\frac{-1}{2 \Delta_{0}}+\frac{q^{2}-(v \cdot q)^{2}}{\Delta_{0}^{2}}\right) \\
& T_{2}^{(1)}=m_{b} E_{b}\left(\frac{1}{\Delta_{0}}-\frac{2 m_{b}\left(m_{b}-v \cdot q\right)}{\Delta_{0}^{2}}\right)+\frac{2 m_{b}}{3}\left(K_{b}+G_{b}\right)\left(\frac{1}{\Delta_{0}}+\frac{2 m_{b} v \cdot q}{\Delta_{0}^{2}}\right) \\
& T_{3}^{(1)}=-m_{b} E_{b}\left(\frac{m_{b}-v \cdot q}{\Delta_{0}^{2}}\right)-\frac{2 m_{b}}{3}\left(K_{b}+G_{b}\right)\left(\frac{m_{b}-v \cdot q}{\Delta_{0}^{2}}\right) \\
& T_{4}^{(1)}=\frac{2 m_{b}}{3}\left(K_{b}+G_{b}\right)\left(\frac{2}{\Delta_{0}^{2}}\right) \\
& T_{5}^{(1)}=m_{b} E_{b}\left(\frac{m_{b}-v \cdot q}{\Delta_{0}^{2}}\right)+\frac{2 m_{b}}{3}\left(K_{b}+G_{b}\right)\left(\frac{-m_{b}-v \cdot q}{\Delta_{0}^{2}}\right),
\end{aligned}
$$

where the spin-energy, kinetic energy and total energy matrix elements $G_{b}, K_{b}$ and $E_{b}$, respectively, of the b-quark in the hadron $H_{b}$ are defined as in [ [ 
The order $k^{2}$ contributions are

$$
\begin{aligned}
T_{1}^{(2)} & =-\frac{m_{b}^{2} K_{b}}{3}\left(m_{b}-v \cdot q\right)\left(\frac{4\left(q^{2}-(v \cdot q)^{2}\right)}{\Delta_{0}^{3}}-\frac{3}{\Delta_{0}^{2}}\right) \\
T_{2}^{(2)} & =-\frac{2 m_{b}^{3} K_{b}}{3}\left(\frac{4\left(q^{2}-(v \cdot q)^{2}\right)}{\Delta_{0}^{3}}-\frac{3}{\Delta_{0}^{2}}\right)+\frac{4 m_{b}^{2} K_{b} v \cdot q}{3 \Delta_{0}^{2}} \\
T_{3}^{(2)} & =-\frac{m_{b}^{2} K_{b}}{3}\left(\frac{4\left(q^{2}-(v \cdot q)^{2}\right)}{\Delta_{0}^{3}}-\frac{3}{\Delta_{0}^{2}}\right)+\frac{2 m_{b}^{2} K_{b}}{3 \Delta_{0}^{2}} \\
T_{4}^{(2)} & =0 \\
T_{5}^{(2)} & =\frac{m_{b}^{2} K_{b}}{3}\left(\frac{4\left(q^{2}-(v \cdot q)^{2}\right)}{\Delta_{0}^{3}}-\frac{5}{\Delta_{0}^{2}}\right) .
\end{aligned}
$$

There is an additional gluonic contribution originating from the gluon operator in the second term of (四), i.e.

$$
\frac{g}{2 \Delta_{0}^{2}} \bar{b} G^{\alpha \beta} \epsilon_{\alpha \beta \lambda \sigma}\left(m_{b}-q\right)^{\lambda}\left[g^{\mu \sigma} \gamma^{\nu}+g^{\nu \sigma} \gamma^{\mu}-g^{\mu \nu} \gamma^{\sigma}+i \epsilon^{\mu \sigma \nu \tau} \gamma_{\tau}\right] \frac{1-\gamma_{5}}{2} b
$$

which leads to

$$
\begin{aligned}
T_{1}^{(g)} & =-\frac{m_{b}^{2} G_{b}}{3 \Delta_{0}^{2}}\left(m_{b}-v \cdot q\right) \\
T_{2}^{(g)} & =\frac{2 m_{b}^{3} G_{b}}{3 \Delta_{0}^{2}} \\
T_{3}^{(g)} & =-\frac{m_{b}^{2} G_{b}}{3 \Delta_{0}^{2}} \\
T_{4}^{(g)} & =0 \\
T_{5}^{(g)} & =-\frac{m_{b}^{2} G_{b}}{3 \Delta_{0}^{2}}
\end{aligned}
$$

Summing the various contributions $T_{i}=\sum_{z} T_{i}^{(z)}, i \in\{0,1,2, g\}$ we obtain

$$
\begin{aligned}
T_{1}= & \frac{1}{2 \Delta_{0}}\left(m_{b}-v \cdot q\right)\left(1+X_{b}\right)+\frac{2 m_{b}}{3}\left(K_{b}+G_{b}\right)\left(\frac{-1}{2 \Delta_{0}}+\frac{q^{2}-(v \cdot q)^{2}}{\Delta_{0}^{2}}\right) \\
& +\frac{m_{b} E_{b}}{2 \Delta_{0}}-\frac{m_{b}^{2} G_{b}}{3 \Delta_{0}^{2}}\left(m_{b}-v \cdot q\right)
\end{aligned}
$$




$$
\begin{aligned}
T_{2}= & \frac{m_{b}}{\Delta_{0}}\left(1+X_{b}\right)+\frac{2 m_{b}}{3}\left(K_{b}+G_{b}\right)\left(\frac{1}{\Delta_{0}}+\frac{2 m_{b} v \cdot q}{\Delta_{0}^{2}}\right) \\
& +\frac{m_{b} E_{b}}{\Delta_{0}}+\frac{4 m_{b}^{2} K_{b} v \cdot q}{3 \Delta_{0}^{2}}+\frac{2 m_{b}^{3} G_{b}}{3 \Delta_{0}^{2}} \\
T_{3}= & \frac{1}{2 \Delta_{0}}\left(1+X_{b}\right)-\frac{2 m_{b}}{3}\left(K_{b}+G_{b}\right) \frac{m_{b}-v \cdot q}{\Delta_{0}^{2}}+\frac{2 m_{b}^{2} K_{b}}{3 \Delta_{0}^{2}}-\frac{m_{b}^{2} G_{b}}{3 \Delta_{0}^{2}} \\
T_{4}= & \frac{4 m_{b}}{3 \Delta_{0}^{2}}\left(K_{b}+G_{b}\right) \\
T_{5}= & \frac{-1}{2 \Delta_{0}}\left(1+X_{b}\right)-\frac{2 m_{b}}{3}\left(K_{b}+G_{b}\right) \frac{2 m_{b}+v \cdot q}{\Delta_{0}^{2}}+\frac{m_{b}^{2} G_{b}}{3 \Delta_{0}^{2}}
\end{aligned}
$$

where

$$
X_{b}=\frac{-2\left(m_{b}-v \cdot q\right) m_{b} E_{b}}{\Delta_{0}}-\frac{8 m_{b}^{2} K_{b}}{3 \Delta_{0}^{2}}\left(q^{2}-(v \cdot q)^{2}\right)+\frac{2 m_{b}^{2} K_{b}}{\Delta_{0}}
$$

Our formulae for $T_{1}, T_{2}, T_{3}$ coincide with the ones given in [4, whereas the $T_{4}$ and $T_{5}$ expressions are new.

We get the resulting differential decay rate (14) by taking the imaginary part of $T^{\mu \nu}$ and dividing it by $(-\pi)$. This amounts to the replacements

$$
\begin{aligned}
& \frac{1}{\Delta_{0}} \rightarrow \delta\left(\left(m_{b} v-q\right)^{2}-m_{j}^{2}\right), \\
& \frac{1}{\Delta_{0}^{2}} \rightarrow-\delta^{\prime}\left(\left(m_{b} v-q\right)^{2}-m_{j}^{2}\right), \\
& \frac{1}{\Delta_{0}^{3}} \rightarrow \frac{1}{2} \delta^{\prime \prime}\left(\left(m_{b} v-q\right)^{2}-m_{j}^{2}\right) .
\end{aligned}
$$

From the dispersion relation in the variable $v . q$ it can be seen [4] that this replacement is only legitimate if one integrates over the whole neutrino phase space. This is no restriction as the $\tau$-neutrino is not detected.

As we are only interested in the double differential rate with respect to $q^{2}$ and $E_{\tau}$ we integrate over $E_{\nu}$. Taking into account the $\delta$-function structure according to $(22)$ the integral over $E_{\nu}$ takes the following form

$$
\int_{E_{\nu}^{\min }\left(q^{2}, E_{\tau}\right)}^{E_{\nu}^{\max }\left(q^{2}, E_{\tau}\right)} \mathrm{d} E_{\nu}\left[f_{1} \delta\left(E_{\nu}-E_{\nu}^{0}\right)+f_{2} \delta^{\prime}\left(E_{\nu}-E_{\nu}^{0}\right)+f_{3} \delta^{\prime \prime}\left(E_{\nu}-E_{\nu}^{0}\right)\right],
$$


where

$$
E_{\nu}^{0}=\frac{m_{b}^{2}-m_{j}^{2}+q^{2}}{2 m_{b}}-E_{\tau} .
$$

The limits of integration in (23) are as follows: i) for the values of $\left(q^{2}, E_{\tau}\right)$ lying inside of region A of Fig.1 (for which the minimum mass of the final hadronic state coincides with $M_{X_{m i n}}$, the mass of the lightest hadron state containing a quark $j$ ),

$$
\begin{aligned}
E_{\nu}^{\min } & =\frac{q^{2}-m_{\tau}^{2}}{2\left(E_{\tau}+\sqrt{E_{\tau}^{2}-m_{\tau}^{2}}\right)} \\
E_{\nu}^{\max } & =\frac{M_{H_{b}}^{2}-M_{X_{\min }}^{2}+q^{2}}{2 M_{H_{b}}}-E_{\tau} ;
\end{aligned}
$$

ii) for the values of $\left(q^{2}, E_{\tau}\right)$ inside of region B of Fig.1,

$$
\begin{aligned}
E_{\nu}^{\text {min }} & =\frac{q^{2}-m_{\tau}^{2}}{2\left(E_{\tau}+\sqrt{E_{\tau}^{2}-m_{\tau}^{2}}\right)} \\
E_{\nu}^{\max } & =\frac{q^{2}-m_{\tau}^{2}}{2\left(E_{\tau}-\sqrt{E_{\tau}^{2}-m_{\tau}^{2}}\right)} .
\end{aligned}
$$

The continuous line which separates the two regions A and B is given by

$$
q^{2}=\frac{M_{H_{b}}\left(M_{H_{b}}^{2}-M_{X_{m i n}}^{2}+m_{\tau}^{2}-2 M_{H_{b}} E_{\tau}\right)\left(E_{\tau}-\sqrt{E_{\tau}^{2}-m_{\tau}^{2}}\right)+m_{\tau}^{2} M_{X_{\min }}^{2}}{M_{H_{b}}^{2}+m_{\tau}^{2}-2 M_{H_{b}} E_{\tau}} .
$$

As in the massless lepton case, the actual particle masses are so that always $E_{\nu}^{0}<E_{\nu}^{\max }$ as long as $\left(q^{2}, E_{\tau}\right)$ stay within region A of the Dalitz plot. This is however no longer true for region $B$, where this inequality has to be imposed explicitly. Its effect is to give an upper bound on the mass of the final hadronic state (smaller than the kinematically allowed one $\left(M_{H_{b}}-m_{\tau}\right)$ ). This is a new aspect which was not present in the massless lepton case.

The net result of all the restrictions imposed by the $\delta$ functions and its derivatives is that the double differential decay rate (23) is nonvanishing only within the partonic region delimited by the interrupted line in Fig.1, 
determined in terms of quark masses. Its expression is

$$
\begin{aligned}
& \quad \frac{d \Gamma}{\Gamma_{b} d y d \hat{q}^{2}}= \\
& \Theta\left(z_{+}\right) \Theta\left(-z_{-}\right)\left(12\left(-\hat{q}^{4}+2 \hat{q}^{2} y+\hat{q}^{2} \rho-\hat{q}^{2} \eta-\hat{q}^{2}-y^{2}-y \rho+y \eta+y+\rho \eta-\eta\right)\right. \\
& +12 E_{b}\left(2 \hat{q}^{4}-2 \hat{q}^{2} y-2 \hat{q}^{2} \rho+2 \hat{q}^{2} \eta+y \rho-y \eta+y-2 \rho \eta\right) \\
& +8 K_{b}\left(-\hat{q}^{4}+\hat{q}^{2} \rho-\hat{q}^{2} \eta+2 \hat{q}^{2}-3 y+\rho \eta+6 \eta\right) \\
& \left.+8 G_{b}\left(2 \hat{q}^{4}-2 \hat{q}^{2} y-2 \hat{q}^{2} \rho+2 \hat{q}^{2} \eta-\hat{q}^{2}+y \rho-y \eta-2 y-2 \rho \eta+5 \eta\right)\right) \\
& -\delta\left(z_{+}\right)\left(24 E_{b} \sigma_{+}\left(-\hat{q}^{2} y-2 \hat{q}^{2} \sigma_{+}+2 \hat{q}^{2}+y^{2}+2 y \sigma_{+}-y \eta-2 y-2 \sigma_{+} \eta+2 \eta\right)\right. \\
& +4 K_{b}\left(\hat{q}^{2} y^{2}+4 \hat{q}^{2} y \sigma_{+}+4 \hat{q}^{2} \sigma_{+}^{2}-20 \hat{q}^{2} \sigma_{+}-4 \hat{q}^{2} \eta-y^{3}+y^{2} \eta+4 y \sigma_{+}^{2}+4 y \sigma_{+} \eta\right. \\
& \left.\quad+12 y \sigma_{+}+4 y \eta+4 \sigma_{+}^{2} \eta-20 \sigma_{+} \eta-4 \eta^{2}\right) \\
& +16 G_{b}\left(\hat{q}^{4}-\hat{q}^{2} y \sigma_{+}-\hat{q}^{2} y-2 \hat{q}^{2} \sigma_{+}^{2}+\hat{q}^{2} \sigma_{+}-\hat{q}^{2}+y^{2} \sigma_{+}+2 y \sigma_{+}^{2}-y \sigma_{+} \eta+y \sigma_{+}\right. \\
& \left.\left.\quad+y \eta-2 \sigma_{+}^{2} \eta-\sigma_{+} \eta-\eta^{2}+\eta\right)\right) \\
& +\delta\left(z_{-}\right)\left(24 E_{b} \sigma_{-}\left(-\hat{q}^{2} y-2 \hat{q}^{2} \sigma_{-}+2 \hat{q}^{2}+y^{2}+2 y \sigma_{-}-y \eta-2 y-2 \sigma_{-} \eta+2 \eta\right)\right. \\
& +4 K_{b}\left(\hat{q}^{2} y^{2}+4 \hat{q}^{2} y \sigma_{-}+4 \hat{q}^{2} \sigma_{-}^{2}-20 \hat{q}^{2} \sigma_{-}-4 \hat{q}^{2} \eta-y^{3}+y^{2} \eta+4 y \sigma_{-}^{2}+4 y \sigma_{-} \eta\right. \\
& \left.\quad+12 y \sigma_{-}+4 y \eta+4 \sigma_{-}^{2} \eta-20 \sigma_{-} \eta-4 \eta^{2}\right) \\
& +16 G_{b}\left(\hat{q}^{4}-\hat{q}^{2} y \sigma_{-}-\hat{q}^{2} y-2 \hat{q}^{2} \sigma_{-}^{2}+\hat{q}^{2} \sigma_{-}-\hat{q}^{2}+y^{2} \sigma_{-}+2 y \sigma_{-}^{2}-y \sigma_{-} \eta+y \sigma_{-}\right. \\
& \left.\left.\quad+y \eta-2 \sigma_{-}^{2} \eta-\sigma_{-} \eta-\eta^{2}+\eta\right)\right) \\
& +8 \delta^{\prime}\left(z_{+}\right) \sigma_{+} K_{b}\left(4 \hat{q}^{4}-\hat{q}^{2} y^{2}-4 \hat{q}^{2} y \sigma_{+}-4 \hat{q}^{2} y-4 \hat{q}^{2} \sigma_{+}^{2}+4 \hat{q}^{2} \eta+y^{3}+4 y^{2} \sigma_{+}\right. \\
& \left.\quad-y^{2} \eta+4 y \sigma_{+}^{2}-4 y \sigma_{+} \eta-4 \sigma_{+}^{2} \eta\right) \\
& -8 \delta^{\prime}\left(z_{-}\right) \sigma_{-} K_{b}\left(4 \hat{q}^{4}-\hat{q}^{2} y^{2}-4 \hat{q}^{2} y \sigma_{-}-4 \hat{q}^{2} y-4 \hat{q}^{2} \sigma_{-}^{2}+4 \hat{q}^{2} \eta+y^{3}+4 y^{2} \sigma_{-}\right. \\
& \left.\quad-y^{2} \eta+4 y \sigma_{-}^{2}-4 y \sigma_{-} \eta-4 \sigma_{-}^{2} \eta\right)
\end{aligned}
$$

where

$$
\begin{gathered}
z_{ \pm}=1+\hat{q}^{2}-\rho-y-2 \sigma_{ \pm}, \quad \sigma_{ \pm}=\left(\hat{q}^{2}-\eta\right) \xi_{ \pm}, \quad \xi_{ \pm}=\frac{1}{y \pm \sqrt{y^{2}-4 \eta}} \\
y=\frac{2 E_{\tau}}{m_{b}}, \quad \hat{q}^{2}=\frac{q^{2}}{m_{b}^{2}}, \quad \rho=\frac{m_{j}^{2}}{m_{b}^{2}}, \quad \eta=\frac{m_{\tau}^{2}}{m_{b}^{2}}
\end{gathered}
$$


and

$$
\Gamma_{b}=\frac{\left|V_{b j}\right|^{2} G_{F}^{2} m_{b}^{5}}{192 \pi^{3}} .
$$

In order to obtain the lepton spectrum, the double differential decay rate (30) has to be integrated over $q^{2}$. The integration limits are

$$
\hat{q}_{ \pm}^{2}=\frac{(1-\rho+\eta-y)\left(y \pm \sqrt{y^{2}-4 \eta}\right)+2 \rho \eta}{2(1+\eta-y)}
$$

The upper limit $\hat{q}_{+}^{2}$ corresponds to $z_{+}=0$ and the lower one $\hat{q}_{-}^{2}$ to $z_{-}=0$. The result is

$$
\begin{aligned}
& \frac{d \Gamma}{\Gamma_{b} d y}= \\
& \sqrt{y^{2}-4 \eta}\left(\left[2\left(-2 y^{2}+3 y \eta+3 y-4 \eta\right)-6 y \rho-\frac{6(-4 \eta+y+y \eta)}{(1+\eta-y)^{2}} \rho^{2}\right.\right. \\
& \left.+\frac{-16 \eta+6 y+6 \eta y-2 y^{2}}{(1+\eta-y)^{3}} \rho^{3}\right] \\
& +E_{b}\left[4\left(-y^{2}+3 y-2 \eta\right)+\frac{12\left(-6 \eta+2 \eta^{2}+y+3 \eta y-\eta y^{2}\right)}{(1+\eta-y)^{3}} \rho^{2}\right. \\
& \left.+\frac{4\left(20 \eta-4 \eta^{2}-6 y-14 \eta y+4 y^{2}+4 \eta y^{2}-y^{3}\right)}{(1+\eta-y)^{4}} \rho^{3}\right] \\
& +K_{b}\left[-\frac{4}{3}\left(-20 \eta+9 y+2 y^{2}\right)\right. \\
& -\frac{4\left(-20 \eta+32 \eta^{2}+4 \eta^{3}+3 y+2 \eta y-25 \eta^{2} y+2 y^{2}+4 \eta y^{2}+2 \eta^{2} y^{2}-2 y^{3}+\eta y^{3}\right)}{(1+\eta-y)^{4}} \rho^{2} \\
& -\frac{4}{3(1+\eta-y)^{5}}\left(80 \eta-104 \eta^{2}+8 \eta^{3}-18 y-40 \eta y+50 \eta^{2} y+10 y^{2}\right. \\
& \left.\left.+22 \eta y^{2}-8 \eta^{2} y^{2}-5 y^{3}-5 \eta y^{3}+y^{4}\right) \rho^{3}\right]+G_{b}\left[-\frac{4}{3}\left(-20 \eta+15 y+2 y^{2}\right)\right. \\
& -\frac{8\left(4 \eta-3 y-3 \eta y+2 y^{2}\right)}{(1+\eta-y)^{2}} \rho-\frac{4\left(12 \eta+4 \eta^{2}-3 y-15 \eta y+3 y^{2}+2 \eta y^{2}\right)}{(1+\eta-y)^{3}} \rho^{2} \\
& \left.\left.-\frac{8\left(-20 \eta+4 \eta^{2}+6 y+14 \eta y-4 y^{2}-4 \eta y^{2}+y^{3}\right)}{3(1+\eta-y)^{4}} \rho^{3}\right]\right) \text {. }
\end{aligned}
$$


The total decay rate can be obtained by integrating the spectrum formula over the partonic range of values for $y$

$$
2 \sqrt{\eta} \leq y \leq 1-\rho+\eta
$$

with the result

$$
\Gamma=\Gamma_{b}\left[f_{0}(\rho, \eta)+E_{b} f_{E}(\rho, \eta)+K_{b} f_{K}(\rho, \eta)+G_{b} f_{G}(\rho, \eta)\right] .
$$

Here

$$
\begin{aligned}
f_{0}(\rho, \eta)= & \sqrt{R}\left(1-7 \eta-7 \eta^{2}+\eta^{3}-7 \rho+12 \eta \rho-7 \eta^{2} \rho-7 \rho^{2}-7 \eta \rho^{2}+\rho^{3}\right) \\
& -24\left[\eta^{2}\left(1-\rho^{2}\right) \log \frac{1+\eta-\rho-\sqrt{R}}{2 \sqrt{\eta}}+(\rho \leftrightarrow \eta)\right] \\
f_{E}(\rho, \eta)= & \sqrt{R}\left(5-19 \eta+5 \eta^{2}-3 \eta^{3}-19 \rho-4 \eta \rho+21 \eta^{2} \rho+5 \rho^{2}+21 \eta \rho^{2}-3 \rho^{3}\right) \\
& -24\left[\eta^{2}\left(1+3 \rho^{2}\right) \log \frac{1+\eta-\rho-\sqrt{R}}{2 \sqrt{\eta}}+(\rho \leftrightarrow \eta)\right] \\
f_{K}(\rho, \eta)= & 2 \sqrt{R}\left(-3+13 \eta+\eta^{2}+\eta^{3}+13 \rho-4 \eta \rho-7 \eta^{2} \rho+\rho^{2}-7 \eta \rho^{2}+\rho^{3}\right) \\
& +48\left[\eta^{2}\left(1+\rho^{2}\right) \log \frac{1+\eta-\rho-\sqrt{R}}{2 \sqrt{\eta}}+(\rho \leftrightarrow \eta)\right] \\
f_{G}(\rho, \eta)= & 2 \sqrt{R}\left(-1+7 \eta+7 \eta^{2}-\eta^{3}+7 \rho-12 \eta \rho+7 \eta^{2} \rho+7 \rho^{2}+7 \eta \rho^{2}-\rho^{3}\right) \\
& +48\left[\eta^{2}\left(1-\rho^{2}\right) \log \frac{1+\eta-\rho-\sqrt{R}}{2 \sqrt{\eta}}+(\rho \leftrightarrow \eta)\right]
\end{aligned}
$$

where

$$
R=(1+\eta-\rho)^{2}-4 \eta
$$

The limit of a massless final state quark $\rho \rightarrow 0$ requires a special treatment because of the appearance of $\delta(y-1-\eta)$ and $\delta^{\prime}(y-1-\eta)$ functions in the lepton spectrum. They are due to the fact that in this limit $z_{+}$vanishes along the entire line $y=1+\eta$. By considering $z_{+}$as a function of $y$ at fixed $\hat{q}^{2}$, one can write

$$
\begin{aligned}
\delta\left(z_{+}\right) & =\frac{1-\eta}{1-\hat{q}^{2}} \delta(y-1-\eta)+\cdots \\
\delta^{\prime}\left(z_{+}\right) & =\frac{(1-\eta)\left(1-\xi_{+} y\right)}{\left(1-\hat{q}^{2}\right)\left[-1+\xi_{+} y+2 \xi_{+}^{2}\left(\hat{q}^{2}-\eta\right)\right]} \delta^{\prime}(y-1-\eta)+\cdots
\end{aligned}
$$


Inserting these relations into Eq.(30) and performing the $\hat{q}^{2}$-integration between the limits

$$
\hat{q}_{ \pm}^{2}=\frac{1}{2}\left(y \pm \sqrt{y^{2}-4 \eta}\right)
$$

one obtains

$$
\begin{aligned}
& \frac{d \Gamma}{\Gamma_{b} d y}=\sqrt{y^{2}-4 \eta}\left[2\left(-2 y^{2}+3 y \eta+3 y-4 \eta\right)\right. \\
& +4 E_{b}\left(-y^{2}+3 y-2 \eta+\frac{1}{2}(1-\eta)^{3} \delta(y-1-\eta)\right) \\
& -\frac{4}{3} K_{b}\left(-20 \eta+9 y+2 y^{2}+(1-\eta)^{2}(5-2 \eta) \delta(y-1-\eta)\right) \\
& \left.-\frac{4}{3} G_{b}\left(-20 \eta+15 y+2 y^{2}-(1-\eta)^{2}(4-\eta) \delta(y-1-\eta)\right)\right] \\
& +8 K_{b}(1-\eta) h(y, \eta) \delta^{\prime}(y-1-\eta) .
\end{aligned}
$$

Here $h(y, \eta)$ is defined by

$$
\begin{aligned}
h(y, \eta)= & \xi_{+}\left(1-\xi_{+} y\right) \int_{\hat{q}_{-}^{2}(y)}^{\hat{q}_{+}^{2}(y)} \mathrm{d} \hat{q}^{2} \frac{\left(\hat{q}^{2}-\eta\right)}{\left(1-\hat{q}^{2}\right)\left[-1+\xi_{+} y+2 \xi_{+}^{2}\left(\hat{q}^{2}-\eta\right)\right]} \times \\
& \left(4 \hat{q}^{4}-\hat{q}^{2} y^{2}-4 \hat{q}^{2} y \sigma_{+}-4 \hat{q}^{2} y-4 \hat{q}^{2} \sigma_{+}^{2}+4 \hat{q}^{2} \eta+y^{3}\right. \\
& \left.+4 y^{2} \sigma_{+}-y^{2} \eta+4 y \sigma_{+}^{2}-4 y \sigma_{+} \eta-4 \sigma_{+}^{2} \eta\right)
\end{aligned}
$$

and satisfies

$$
\lim _{y \rightarrow 1+\eta} \frac{\partial}{\partial y} h(y, \eta)=-\frac{2}{3}(1-\eta)^{2} .
$$

An integration of the spectrum (45) over $y$ within the limits $2 \sqrt{\eta} \leq y \leq 1+\eta$ gives the total rate

$$
\Gamma=\Gamma_{b}\left[g_{0}(\eta)+E_{b} g_{E}(\eta)+K_{b} g_{K}(\eta)+G_{b} g_{G}(\eta)\right]
$$

with

$$
\begin{aligned}
g_{0}(\eta) & =1-8 \eta+8 \eta^{3}-\eta^{4}-12 \eta^{2} \log \eta \\
g_{E}(\eta) & =5-24 \eta+24 \eta^{2}-8 \eta^{3}+3 \eta^{4}-12 \eta^{2} \log \eta \\
g_{K}(\eta) & =-6+32 \eta-24 \eta^{2}-2 \eta^{4}+24 \eta^{2} \log \eta \\
g_{G}(\eta) & =-2+16 \eta-16 \eta^{3}+2 \eta^{4}+24 \eta^{2} \log \eta .
\end{aligned}
$$


This is the same as what one would obtain by directly taking the limit $\rho \rightarrow 0$ in (36). In doing the $y$-integration of (45), the end-point $y=1+\eta$ must be considered as included in the region of integration.

Before plotting the lepton energy spectrum we need to specify the values of the nonperturbative matrix elements $G_{b}$ and $K_{b}$. The matrix element $E_{b}$ is given by $E_{b}=G_{b}+K_{b}$. $G_{b}$ is related to the hyperfine splitting of degenerate heavy meson partners and is taken as $G_{b}=-0.0065$ as in 4 . For $K_{b}$ we take $K_{b}=0.01$. Further parameters are $m_{b}=4.8 \mathrm{GeV}$ and $m_{c}=1.39 \mathrm{GeV}$. We shall plot the energy spectrum for mesonic b-decays into $\mathrm{c}$ and $\mathrm{u}$.

Let us first discuss the decay spectrum (34) for $b \rightarrow c$ plotted in Fig.3. The leading order contribution corresponding to free quark decay is larger than the $O\left(1 / m_{b}^{2}\right)$ corrected spectrum over most of the $y$-range. The corrections become larger as the lepton energy increases. The corrected spectrum takes an upward turn to the high end of the spectrum. The OPE can, however, no longer be trusted in local sense at the high end of the spectrum. As a rough estimate where this happens we demand that the nonperturbative terms should not exceed $30 \%$ of the leading parton model result. Judging from the curve in Fig.3 this means that we trust the energy spectrum locally up to $y \lesssim 0.98$. However, assuming duality, the integrated spectrum is expected to be given correctly even including the high end of the spectrum.

The total inclusive decay rate $B \rightarrow X_{c} \tau \bar{\nu}_{\tau}$ is

$$
\Gamma / \Gamma_{b}=\left(0.122+1.286 \cdot E_{b}-1.409 \cdot K_{b}-0.245 \cdot G_{b}\right)=0.122-0.008
$$

for $m_{\tau}=1.78 \mathrm{GeV}, m_{j}=m_{c}=1.39 \mathrm{GeV}$ and $m_{b}=4.8 \mathrm{GeV}$, whereas for $m_{\tau}=0$ we would have had

$\Gamma_{m_{\tau}=0} / \Gamma_{b}=\left(0.543+3.361 \cdot E_{b}-3.904 \cdot K_{b}-1.086 \cdot G_{b}\right)=0.543-0.020$.

The lepton mass effects appear thus to increase the procentual contribution of the nonperturbative $1 / m_{b}^{2}$ corrections in the total result from $-3.7 \%$ to about $-6.6 \%$.

Next turn to the tau spectrum for $b \rightarrow u$, which is plotted in Fig.4. Also shown is the free quark decay spectrum for comparison. Again one should trust the spectrum locally only for $y \lesssim 0.98$.

The total decay rate is

$$
\Gamma / \Gamma_{b}=\left(0.371+2.584 \cdot E_{b}-2.954 \cdot K_{b}-0.741 \cdot G_{b}\right)=0.371-0.016,
$$


where the value $m_{u}=0$ has been taken.

While preparing this manuscript for publication we became aware of the paper of Koyrakh [10], who has also considered lepton mass effects within the operator product expansion method. While we agree with his final numerical results, we do not agree with all his formulas, which we suspect to be marred with misprints.

\section{References}

[1] J.Chay, H.M.Georgi and B.Grinstein, Phys.Lett. B247 (1990) 399

[2] I.I. Bigi, M. Shifman, N.G. Uraltsev and A.I. Vainshtein, Phys. Rev. Lett. 71(1993)496

[3] B. Blok, L. Koyrakh, M. Shifman and A.I. Vainshtein, preprint NSFITP-93-68, TPI-MINN-93/33-T, [hep/ph 9307247]

[4] A.V. Manohar and M.B. Wise, CALT-68-1883, hep-ph/9308246

[5] T. Mannel, Darmstadt preprint IKDA 93/26, hep-ph/9308262

[6] I. Tomalin ALEPH: Talk given at the International Europhysics Conference on High Energy Physics. Marseille (1993); A. Putzer, ALEPH: Talk given at the 5th International Symposium on Heavy Flavour Physics. Montreal, Canada (1993), Heidelberg preprint HD-IHEP/93-03

[7] V.A. Novikov, M.A. Shifman, A.I. Vainshtein and V.I. Zakharov, Fortschr.Phys. 32(1984)585

[8] J.G. Körner and G. Thompson, Phys. Lett. B264(1991)185

[9] J.G. Körner and G.A. Schuler, Z. Phys. C 46(1990)93

[10] L. Koyrakh, TPI-MINN-93/47-T, [hep/ph 9311215] 


\section{Figure Captions}

Fig.1 The Dalitz plot for the inclusive decay $B \rightarrow X_{c} \tau \bar{\nu}_{\tau}$. The interrupted line shows the region of the phase space which is populated in the parton model.

Fig.2 The Dalitz plot for the inclusive decay $B \rightarrow X_{u} \tau \bar{\nu}_{\tau}$. The interrupted line shows the region of the phase space which is populated in the parton model.

Fig.3 $\tau$-energy spectrum for inclusive semileptonic bottom meson decays $b \rightarrow c$, normalized through division by $\Gamma_{b}$. The continuous line is the spectrum including $O\left(1 / m_{b}^{2}\right)$ corrections and the dotted line shows the leading parton model result.

Fig.4 $\tau$-energy spectrum for inclusive semileptonic bottom meson decays $b \rightarrow u$, normalized through division by $\Gamma_{b}$. The continuous line is the spectrum including $O\left(1 / m_{b}^{2}\right)$ corrections and the dotted line shows the leading parton model result. 


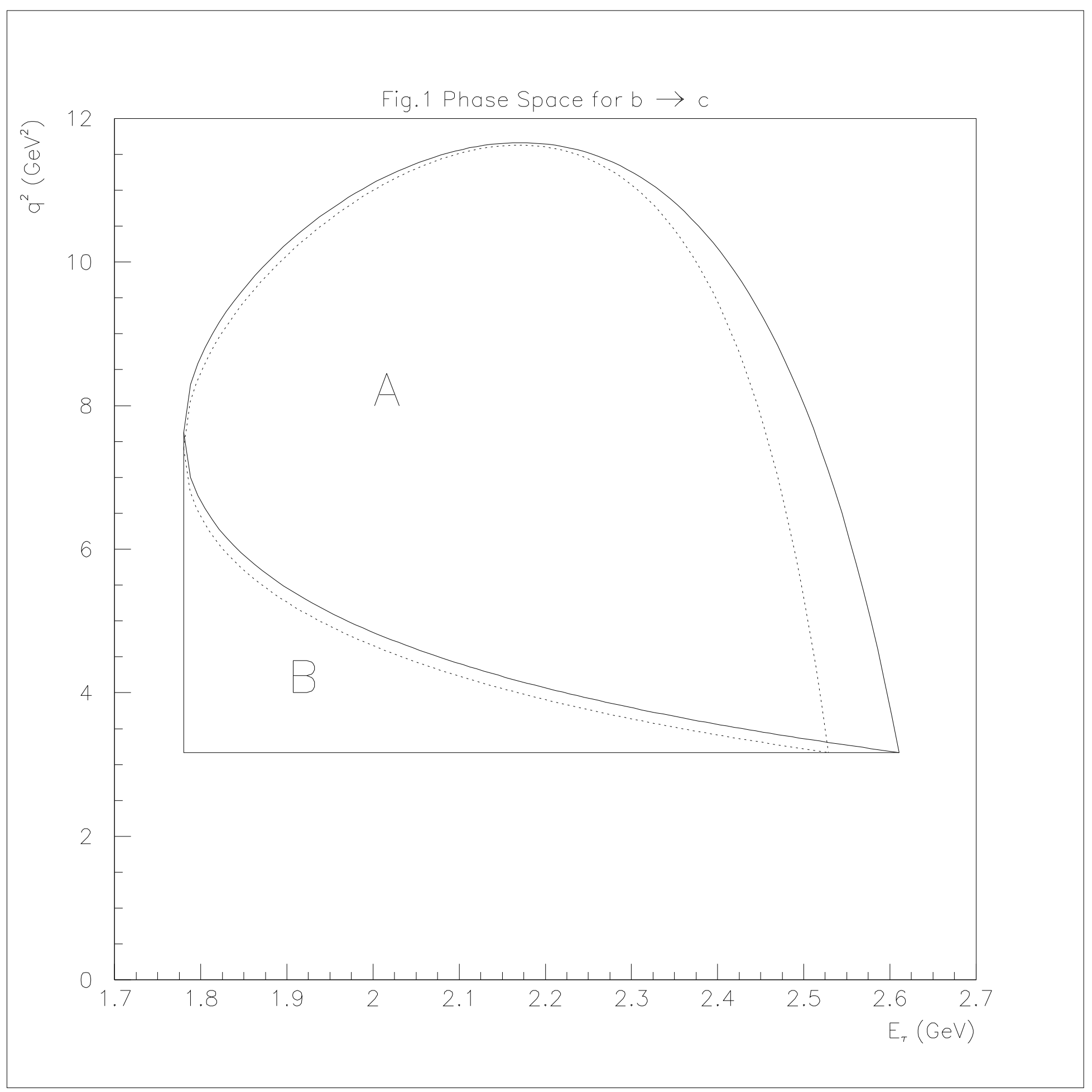




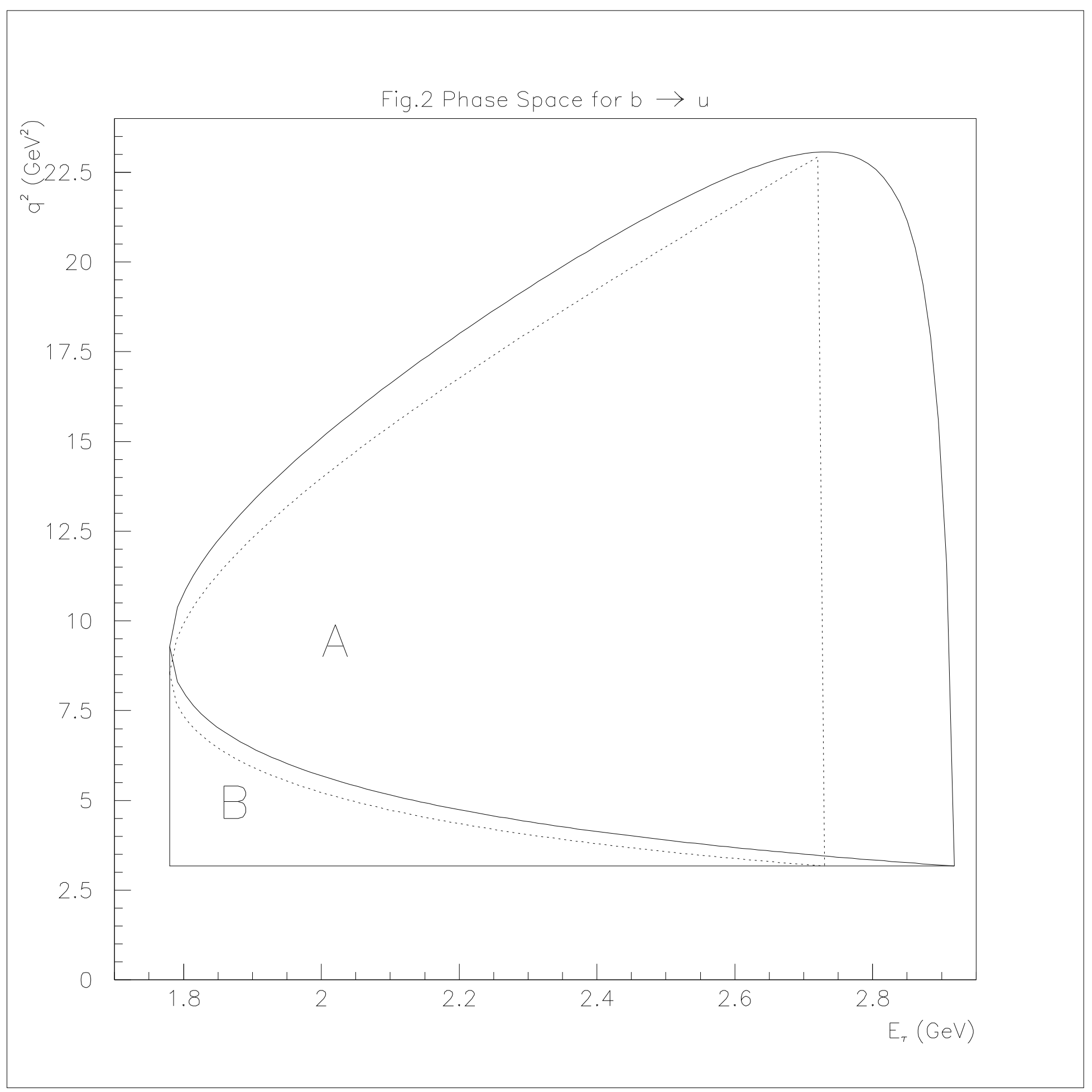




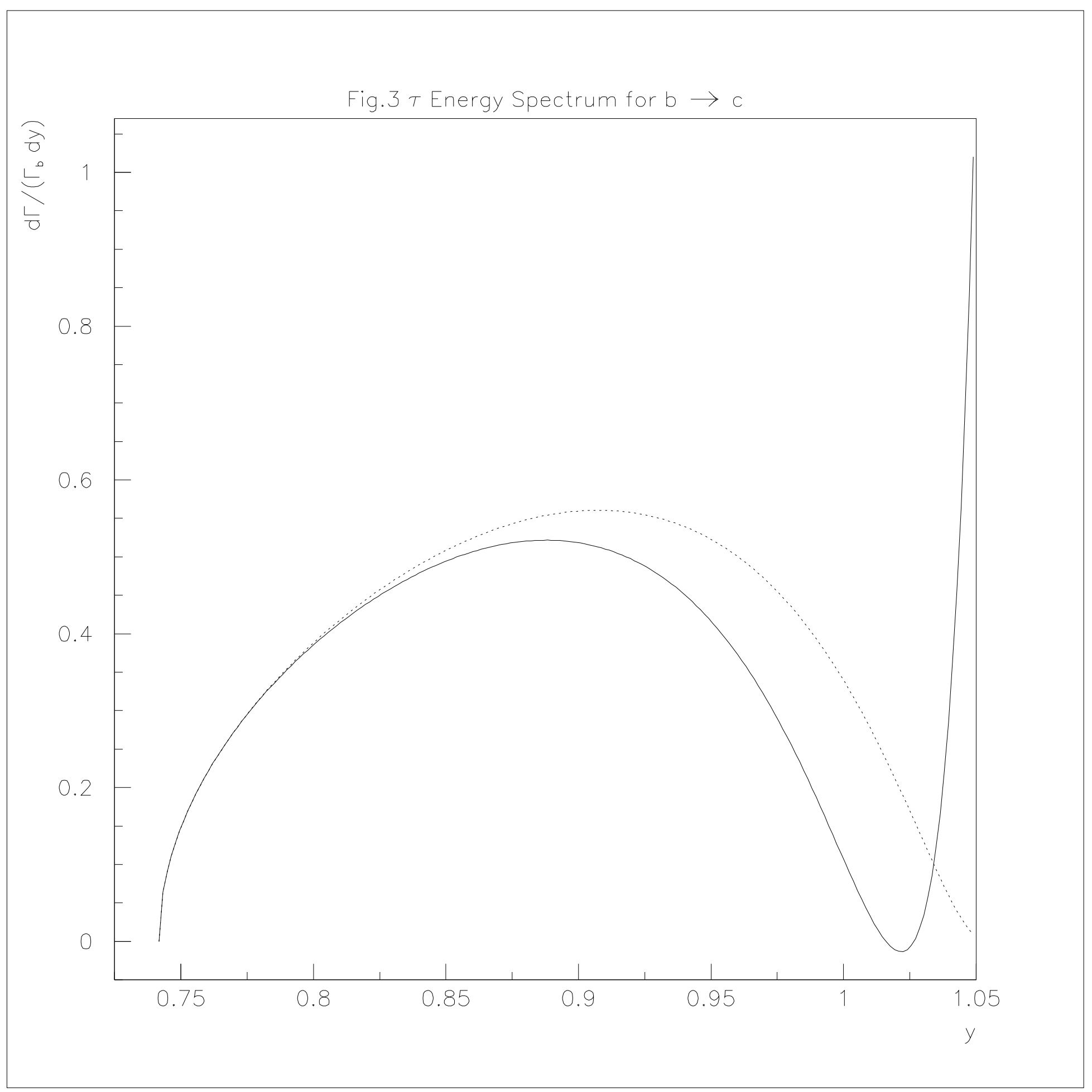




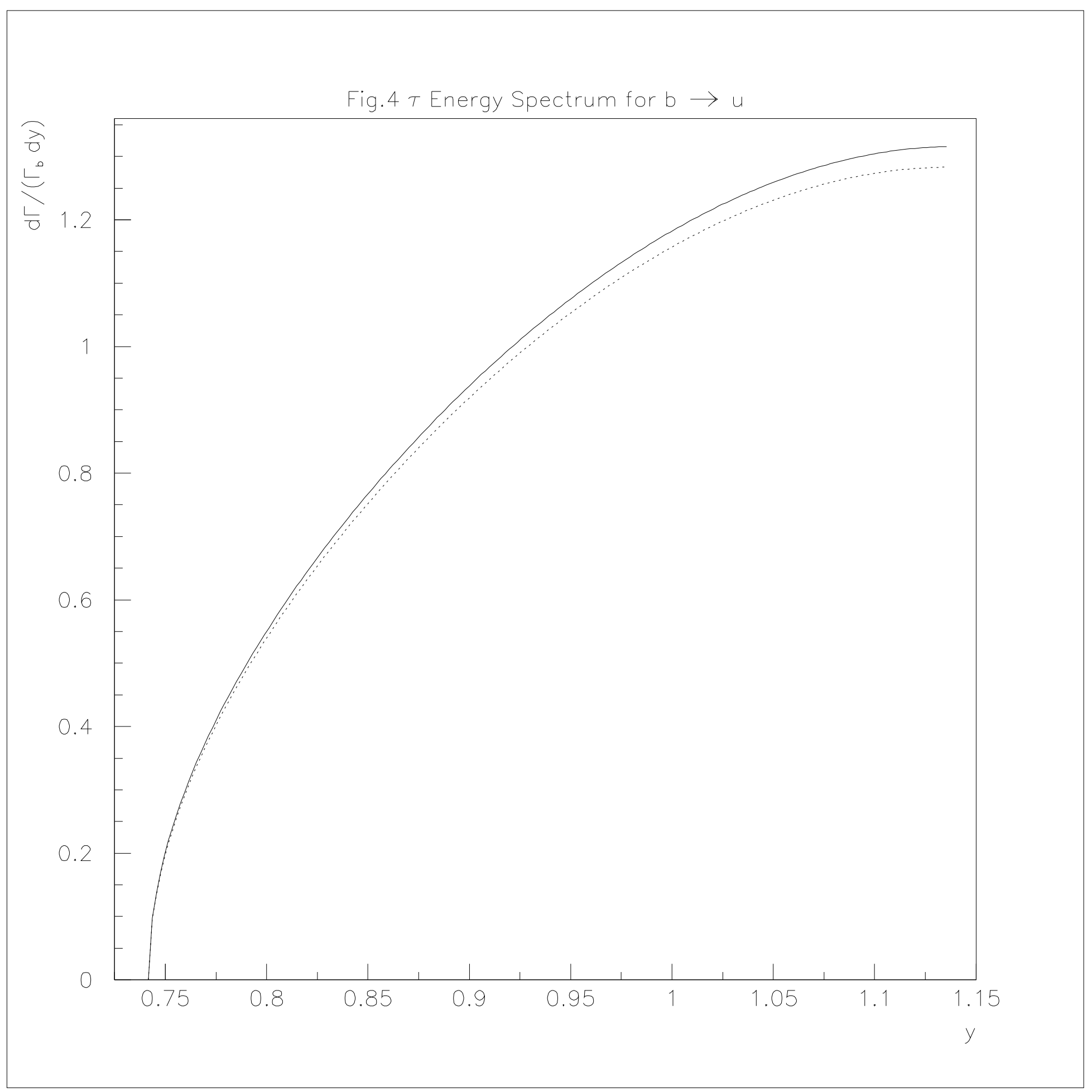

